\title{
El reto medioambiental en la Europa oriental: Lecciones de la experiencia occidental *
}

\section{Susana Aguilar Fernández * Brian Slocock ***}

El término 'déficit de implementación' ha sido utilizado por analistas políticos para describir la distancia existente entre los objetivos recogidos en distintos programas de políticas públicas y los resultados realmente conseguidos. En Alemania fue el Consejo de Expertos para Cuestiones Medioambientales el que primeramente, en 1974, acuñó este término (Vollzugsdefizit) para aplicarlo a la política de protección del medio ambiente. En esta política el déficit de implementación definía una situación en la que, a pesar de existir una legislación comprehensiva y exigente, predominaba un cumplimiento insatisfactorio de los objetivos políticos. Este déficit habría conducido a que, a pesar de los esfuerzos legislativos, la degradación medioambiental no (hubiera sido] reducida en la medida esperada. (HUCKE y ULLMANN, 1980: 105). En un principio el déficit de implementación se atribuyó al proceso de negociación entre las autoridades y las industrias en la fase previa a la puesta en práctica de la política medioambiental; este proceso favorecía, supuestamente, una aplicación a la baja de la política y consolidaba la posición privilegiada de los grupos industriales (MAYnTz et al., 1978) Posteriormente, se relativizó la relevancia del déficit señalándose que éste no significaba tanto una insuficiente aplicación de la política como la existencia de leyes inadecuadas, y excesivamente estrictas, para ser cumplidas (RANDELZHOFER y WILKE, 1981). Asimismo, se admitió que la negociación, que teóricamente acompañaba al déficit, suponía un intento de corrección de una política elaborada erróneamente, así como la incorporación de intereses olvidados durante la formulación de los programas medioambientales (MAYNTZ, 1980).

A pesar de que en su origen el déficit de implementación sirvió para describir la política medioambiental de los países occidentales, el término es aún más adecuado para los antiguos países comunistas del Este de Europa. Este déficit se constata actualmente en los esfuerzos de los nuevos gobiernos democráticos de esta región por superar los graves daños ecológicos heredados del pasado, así como en la preocupación de sus responsables políticos acerca de si, una vez más, se enfrentarán a una frustrante diferencia entre intenciones y resultados o si, por el contrario, podrán reducir este problema a través de nuevas aproximaciones políticas y diseños institucionales.
A la hora de afrontar los problemas medioambientales del Este de Europa se ha producido un considerable esfuerzo por transferir y aplicar la 'sabiduría de Occidente'. Este proceso, que ha sido llevado a cabo conjuntamente por agencias públicas de distintos países y por ONGs, ha adoptado dos formas: Una general a través del consejo político y, otra más particular, a través de diversos programas de apoyo. Frecuentemente ha existido una tendencia a ofrecer (y de manera paralela a aceptar acríticamente) la experiencia occidental como modelo a imitar.

De este modo, se han infraestimado las especificidades de los contextos nacionales del Este, las posibilidades de nuevas vías para la creación institucional, y los límites de las organizaciones de Occidente. Este artículo intentará aproximarse a algunos de estos problemas a través de un doble prisma: La revisión de la naturaleza de los problemas medioambientales en el Este de Europa, y el análisis crítico de la actuación de algunos países occidentales -España, Gran Bretaña, y Alemania - en la política de control de la contaminación atmosférica. El objetivo final será identificar lo que el Este puede aprender de la experiencia de Occidente, tanto en su vertiente de fracaso como de éxito.

\section{Los rasgos comunes de la política medioambiental}

Existe un debate dentro de los análisis sobre políticas públicas entre la 'hipótesis de la especificidad de la política pública' (Lowl, 1964) y la 'hipótesis del estilo político nacional' (RICHARDSON, 1982): La primera defiende que las distintas políticas están determinadas básicamente por sus características inherentes y que, por lo tanto, a nivel comparativo éstas tienden a ser similares con independencia del país objeto de análisis '; la segunda sugiere que las políticas son diferentes en cada país debido a los específicos contextos históricos y tradiciones 
institucionales nacionales. Cada una de estas aproximaciones ha encontrado tanto detractores como defensores en el caso de la política medioambiental aunque no deberían, en principio, ser siempre tratadas como excluyentes: Es cierto que las políticas conservacionistas comparten importantes rasgos al margen de los países en los que se desarrollan pero, al mismo tiempo, también es posible identificar en ellas peculiariedades nacionales significativas (como se verá más adelante).

Con relación a los rasgos comunes, se ha señalado que la política de ‘control de la contaminación está sujeta, necesariamente, a una negociación continua entre los inspectores Ipúblicos medioambientales] y la industria" (Vogel, 1986: 64). Por ello, y con independencia del país analizado, parecen existir pautas similares de relación entre los gobiernos, los intereses industriales (business), los grupos medioambientales y los ciudadanos (the public). De esta forma, los grupos industriales suelen disfrutar de una posición privilegiada dentro del proceso político, mientras que otros intereses sociales permanecen relativamente excluidos del mismo. Sin embargo, al mismo tiempo, y en respuesta a presiones internacionales (principalmente de la Unión Europea, UE) y nacionales (de movimientos ecologistas en ascenso) en favor de una política medioambiental más rigurosa y abierta a la participación social, distintos gobiernos han promovido una serie de cambios en sus aproximaciones a esta política aunque, en la mayoría de los casos, los principios fundamentales de la misma se han mantenido prácticamente inalterados (AGUILAR, 1994). Otra peculiaridad de la política medioambiental es que se ha convertido en un área eminentemente técnica en la que los expertos juegan un papel clave; a pesar de ello, los expertos son vistos cada vez con mayor reticencia por parte de los grupos ecologistas y de muchos ciudadanos porque están frecuentemente vinculados a organizaciones industriales.

\section{La situación medioambiental en la Europa del Este}

Como resultado de decenios de desarrollo industrial y gestión económica insensibles al medio ambiente, las emisiones contaminantes en la mayoría de los países de Europa Central y del Este habían alcanzado alrededor de 1989 tal magnitud que la política de protección medioambiental en la región fue pronto considerada un tema de gran importancia a nivel internacional. Esto condujo a la incorporación de programas de asistencia en esta política como componente clave de la mayor parte de los paquetes de ayuda para la región, y a que un amplio abanico de instituciones occidentales - desde la Agencia de Protección Medioambiental de Estados Unidos hasta Greenpeace- lanzasen iniciativas dirigidas a poner a disposición de los responsables políticos del Este de Europa su conocimiento y experiencia en la protección del medio ambiente?

Las razones de la preocupación internacional por el estado medioambiental del Este de Europa resultan bastante evidentes. Por un lado, la contaminación de la región contribuye enormemente a las emisiones transfronterizas europeas que causan la lluvia ácida y al deterioro de los cauces fluviales internacionales y de los ecosistemas marinos. Desde el punto de vista de Occidente, el factor que hasta hace poco tiempo relativizaba la contaminación de la región era que los vientos la empujaban hacia el Este. Pero esto ha dejado de ser ya un consuelo en el escenario internacional posterior a la guerra fria -escenario de 'la casa común europea' y de la preocupación por el impacto global de las emisiones atmosféricas. Por otro lado, estas emisiones han provocado que territorios enteros del Este se vean dramáticamente afectados por una contaminación aguda y generalizada (tanto en el aire, como en el agua y en el suelo) que constituye una amenaza para la salud y el bienestar públicos. La resolución de estas crisis localizadas es así considerada como uno de los problemas sociales más urgentes de la región.

\begin{tabular}{|l|c|c|c|c|}
\hline \multicolumn{4}{|c|}{ Nivel y tendencias en las emisiones de SO2, 1988} \\
\hline & $\begin{array}{c}\text { Emisiones } \\
\text { de SO2 en } \\
\text { millones } \\
\text { de tons. }\end{array}$ & $\begin{array}{c}\text { \% de } \\
\text { cambio } \\
1980-1987\end{array}$ & $\begin{array}{c}\text { Kg/persona } \\
1988\end{array}$ & $\begin{array}{c}\text { Ranking } \\
\text { europeo } \\
1987^{*}\end{array}$ \\
\hline Checoslovaquia & 2,9 & $-6 \%$ & 183 & 6 \\
\hline Polonia & 4,3 & $+5 \%$ & 111 & 3 \\
\hline Bulgaria & 1,1 & $+3 \%$ & 119 & 11 \\
\hline Hungria & 1,4 & $-13 \%$ & 134 & 9 \\
\hline España & 3,2 & $-3 \%$ & 82 & 5 \\
\hline Reino Unido & 3,7 & $-21 \%$ & 64 & 4 \\
\hline
\end{tabular}

El cuadro compara el nivel y la tendencia en las emisiones de $\mathrm{SO} 2$ de cuatro paises del Este de Europa, poco antes de la desintegración del antiguo orden políico, con cifras del Reino Unido (por entonces, el emisor más importante de SO2 de Europa occidental en términos absolutos) y Espaǹa (el más importante en términos per cápita).

- Los dos páses con más altos niveles de emisión eran la Unión Soviética y Alemania Oriental.

Fuente: Calculado a partir de datos en NowıCKI, 1993.

Como puede verse, Polonia era en 1988 dentro de Europa -y dejando a un lado Alemania- el principal país responsable de las emisiones en toneladas de SO2 (4.3), con unos niveles per cápita (111) 35\% más altos que los de España (82) y ello a pesar de tener un menor desarrollo económico que ésta. Checoslovaquia, a su vez, emitía más del doble de la cantidad de SO2 per cápita (183) que el peor caso en Europa Occidental. Además, los únicos países que todavía experimentaban una tendencia creciente en las emisiones de $\mathrm{SO} 2$ en este período estaban en Europa del Este: Polonia $(+5)$ y Bulgaria $(+3)$. 
Si se analiza la contribución de diferentes países europeos a la contaminación atmosférica transfronteriza puede conseguirse una idea aún más precisa de la situación medioambiental en el Este de Europa.

\begin{tabular}{|c|c|}
\hline \multicolumn{2}{|c|}{$\begin{array}{l}\text { Contribuciones a la contaminación atmosférica } \\
\text { transfronteriza. Deposición anual de azufre en paises } \\
\text { vecinos a partir de fuentes nacionales }\end{array}$} \\
\hline & Equivalente a toneladas de azufre \\
\hline 1. Alemania & 9.858 \\
\hline 2. Polonia & 7.955 \\
\hline 3. República Checa & 4.284 \\
\hline 4. Reino Unido & 3.388 \\
\hline 5. Ucrania & 3360 \\
\hline 6. Hungria & 2.214 \\
\hline 7. Francia & 2.043 \\
\hline 8. Rumania & 2.037 \\
\hline 9. Italia & 1.612 \\
\hline 10. Bulgaria & 1.451 \\
\hline
\end{tabular}

- Se excluye la deposición en el mar.

Fuente: Calculado a partir de datos en Ochrona Srodowiska, 1993.

A través de estos datos se comprueba que, de los diez países principalmente responsables de la contaminación transfronteriza, seis son del Este de Europa y de Europa Central -incluyendo aquellos que están en el segundo y tercer lugar, ya que la primera posición, que corresponde a Alemania, se explica básicamente como resultado de las emisiones de Alemania Oriental-. Esta situación explica que muchos especialistas occidentales hayan concluido que la forma más efectiva de reducir las emisiones globales en Europa es a través de programas de descontaminación en el Este y no a través de costosas medidas en Occidente (Ap Simon y WarRen, 1993).

Bajando ya a un nivel más local, el Este de Europa presenta un aspecto muy variado: Hay muchas áreas enormemente degradadas pero también grandes ecosistemas naturales no afectados por la contaminación. En términos generales, la región puede dividirse en dos grupos. Por un lado, están aquellos países que tienen graves problemas medioambientales a nivel agregado y que sufren de una contaminación aguda en todos los medios - Polonia y la República Checa - Por otro lado, y dentro del segundo grupo, se encuentran aquellos países que tienen problemas medioambientales menos serios a nivel agregado -Hungría, Bulgaria, Rumania, y Eslovaquia-. Sin embargo, y con independencia de la imagen que ofrecen las estadísticas, la industrialización forzosa y la irracional planificación urbanística, junto a políticas medioambientales ineficaces, han producido situaciones locales de ecosistemas agudamente contaminados en todos los países del Este. En aquéllos más afectados por este problema existen áreas locales que forman regiones enteras de crisis medioambiental, como en Polonia y en la antigua Checoslovaquia. En este segundo caso las autoridades destacaron seis regiones 'severamente dañadas en su medio ambiente' que cubrían el $7 \%$ del país pero incluían al $57 \%$ de la población -tres de estas regiones son Praga, y las zonas industriales de Bohemia del Norte y Ostrava-Karvina en el norte de Moravia-. En Polonia, por otro lado, existen 27 áreas designadas oficialmente como de 'peligro ecológico', que cubren el $11 \%$ del territorio y abarcan el $35 \%$ de la población; aquí se originan el $77 \%$ de las emisiones de partículas, el $81 \%$ de las emisiones atmosféricas, el $62 \%$ de los residuos líquidos y el $90 \%$ de los sólidos; cinco de ellas - con una población aproximada de 6 millones - son consideradas 'áreas de desastre ecológico', como es el caso del distrito industrial de la Alta Silesia en la provincia de Katowice (principal centro de la industria pesada polaca). Junto a la industria hay que considerar la planificación urbanística que ha situado frecuentemente los hogares de los trabajadores y sus familias de forma cercana a las fuentes de contaminación y, por lo tanto, en peligrosos micro-medioambientes. Las estadísticas de salud pública muestran así una incidencia mucho mayor de mortalidad infantil, malformaciones congénitas, enfermedades respiratorias y cardiovasculares, y cánceres en estas comunidades industriales. La constatación de esta situación no implica que las antiguas autoridades políticas comunistas del Este de Europa ignoraran completamente los problemas medioambientales. Al contrario, el desarrollo de la política medioambiental en la región se produce de forma relativamente paralela al de Occidente (FISHER, 1992; FulleNBACH, 1988; SLOCOCK, 1992). La protección del medio ambiente fue tema frecuente de discusión en los órganos del partido, y dio lugar a medidas legislativas y regulatorias y a programas específicos que intentaron promover tanto la conservación de energía como la reducción de la contaminación ${ }^{3}$. Los gobiernos del Este de Europa se mostraban ansiosos por estar presentes en los distintos forums internacionales medioambientales, y los especialistas de la región jugaron un papel activo en organizaciones internacionales y en redes académicas vinculadas al tema conservacionista ${ }^{4}$. Asimismo, ciertos instrumentos políticos occidentales (como la evaluación de impacto ambiental) fueron rápidamente añadidos al repertorio de prácticas del Este. Pero, a pesar de todo ello, la solución de los problemas medioambientales no gozó de prioridad (como también ocurría en Europa Occidental en aquel momento) porque una acción seria en este sentido habría interferido con la consecución de los objetivos productivistas. Los resultados medioambientales en el Este podrían así calificarse como de dramático déficit de implementación, es decir, como de versión intensificada del fenómeno que se estaba produciendo en Occidente al mismo tiempo. Este déficit es muy claro en el caso de Polonia, país 
que había sido descrito acertadamente como el más sofisticado del Este en cuanto al diseño de su política medioambiental. Con la creación del Ministerio de Medio Ambiente y Recursos Naturales en 1985, Polonia se convirtió en el primer país de la región en dotarse de una institución conservacionista de status ministerial aunque exhibió, a pesar de ello, el peor record medioambiental en ese período (SLOCOCK, 1992; Cummincs, 1992).

\section{Las raíces sistémicas del déficit medioambiental en la Europa del Este}

La razón principal del fracaso de la política medioambiental en el Este de Europa se encuentra en una serie de factores sistémicos que frustraron los esfuerzos de los responsables de la misma por colocar la protección del medio ambiente en las agendas políticas de sus gobiernos. De forma resumida, estos factores sistémicos pueden entenderse desde un punto de vista económico - resultado de las pautas de desarrollo industrial impuestas en la región a lo largo de los últimos cuarenta años-, y político - reflejo de las dinámicas del Estado de partido único estalinista-

Desde finales de los años 40, la Unión Soviética respondió al desarrollo de la guerra fría no sólo consolidando su dominio político sobre el Este de Europa sino también cortando los lazos económicos entre el Este y el Oeste y creando un sistema económico propio y autárquico en torno al Consejo para la Ayuda Económica Mutua (COMECON). Por otra parte Occidente, liderado por Estados Unidos, reaccionó restringiendo también las relaciones con el Este. Dentro del COMECON se estableció una especie de división de trabajo entre Estados, y así el Este de Europa se convirtió en centro de desarrollo de una industria pesada basada en el procesamiento de materias primas y uso de fuentes energéticas soviéticas ${ }^{5}$. Esto condujo a un rápido desarrollo industrial en la región, dirigido frecuentemente según criterios de oportunidad política y de seguridad militar, y planificado con una relativa desconsideración hacia la eficiencia económica, o los costes medioambientales y sociales. Aisladas del más amplio mercado mundial y de fuentes externas de capital, y careciendo de acceso a las modernas tecnologías occidentales, las industrias del Este se caracterizaron durante largo tiempo por ser intensivas en el uso de energía y muy contaminantes -incluso mucho después de que los países occidentales hubieran comenzado a progresar en una dirección más conservacionista-.
Intensidad medioambiental de la producción. Contaminantes por unidad de PIB en Europa del Este y en la Unión Europea en los años $\mathbf{8 0}$

\begin{tabular}{|l|c|c|}
\hline & Este de Europa* & Unión Europea** \\
\hline Emisiones de gas $(\mathrm{kg} / \$ 000)$ & 51 & 24 \\
\hline Emisiones de polvo $(\mathrm{kg} / \$ 000)$ & 13 & 1 \\
\hline Residuos líquidos $(\mathrm{m} 3 / \$ 000)$ & 83 & 24 \\
\hline Residuos sólidos $(\mathrm{kg} / \$ 000)$ & 1.000 & 400 \\
\hline
\end{tabular}

- Media para seis países: Bulgaria, Checoslovaquia, Alemania Oriental, Hungria, Polonia, y Rumania.

* Media para los doce países de la UE

Fuente: ZYLICZ, 1993

Intensidad en el uso de energia en Europa del Este y en la Unión Europea, 1989

\begin{tabular}{|l|c|}
\hline & TOE $/ \$ 000$ PIB \\
\hline UE & 0,25 \\
\hline Bulgaria & 0,58 \\
\hline Checoslovaquia & 0,58 \\
\hline Hungria & 0,38 \\
\hline Polonia & 0,52 \\
\hline Rumania & 0,63 \\
\hline
\end{tabular}

- $\mathrm{TOE}=$ Toneladas de Equivalente de Petróleo.

Fuente: LAPONCHE, 1993.

Como puede verse, la carga medioambiental para generar una unidad de PIB en las economías del Este es mucho mayor que en las de Occidente -probablemente dos o tres veces mayor-, al tiempo que la intensidad energética de las primeras es también superior a la media de la UE.

El deterioro del medio ambiente en el Este fue reforzado asimismo por otro factor clave de naturaleza también sistémica: La dinámica del sistema político implantado en la región bajo la hegemonía soviética. Este sistema tenía diferentes dimensiones, pero la más importante para la presente discusión fue la tendencia a subordinar todas las decisiones sociales a consideraciones de tipo 'productivista'. Este sesgo productivista de los países basados en economías planificadas (o cuasi planificadas), que ha sido a menudo resaltado por los criticos de sus políticas medioambientales, tuvo el efecto de crear una estructura institucional (tanto en las empresas industriales como en los órganos del Estado que las supervisaban) obsesionada por cumplir los objetivos de producción e insensible a los costes -incluyendo aquellos de tipo medioambiental - que acompañaban a este proceso. Todo empresario sabía que era mejor centrar sus distintos recursos en la producción antes que en el control de la contaminación si existía algún riesgo de que los 
objetivos del plan no se cumplieran -riesgo que, además, constituía la norma ya que estos objetivos eran constantemente elevados- Si al centrarse meramente en la producción el empresario entraba en conflicto con las autoridades medioambientales, siempre podía contar con distintos agentes que le protegerían; así, los ministerios económicos e industriales (políticamente más poderosos que las agencias de protección medioambiental) conseguían generalmente negociar exenciones o prórrogas para las regulaciones medioambientales, al tiempo que los órganos del partido podian presionar a las autoridades medioambientales para que éstas ignoraran el asunto; y si todo eso fracasaba, la empresa podía cargar las multas en sus propios presupuestos como coste necesario de la producción.

Muchos analistas han señalado que las economías planificadas centralmente tenían un rasgo inherente contra la protección medioambiental. A pesar de que ese rasgo podría estar presente en los mecanismos de planificación central, no está claro por qué se le habría permitido prevalecer sobre otras instituciones y negar políticas que eran vistas como importantes por las autoridades. El constante socavamiento de los objetivos de protección medioambiental se entiende mejor como producto de la 'crisis de legitimidad' de los regímenes estalinistas, y de su necesidad de poner el énfasis en las recompensas materiales como sustitutivo de la libertad política -lo que se ha denominado pacto autoritario.

\section{Cambios en la Europa del Este: nuevos contextos económicos y políticos}

En los años que han transcurrido desde 1989 ha tenido lugar un amplio abanico de cambios en el Este de Europa, tanto en la esfera económica como en la política. En la esfera política han ido emergiendo sistemas democráticos, basados en elecciones regulares y en competición partidista (a veces muy fragmentada). El cambio económico, por su parte, no ha sido siempre tan rápido como los analistas occidentales propugnaban (y a menudo esperaban), especialmente en la esfera de la privatización de industrias estatales. Pero mientras que los Estados de la región siguen siendo responsables de una parte todavía muy considerable de la economía, la forma en la cual ésta se organiza es ahora muy diferente. En los países que han ido más lejos en el proceso de cambio económico (Polonia, Hungría, y las Repúblicas Checa y Eslovaca), se ha producido una importante reorientación de las relaciones comerciales desde el Este hacia el Oeste, una profunda incorporación a los sistemas internacionales de cambio (algunas monedas son ahora parcialmente con- vertibles), una liberalización de la mayoría de los precios, y una gradual supresión de los subsidios industriales. Asimismo, como ahora es relativamente más fácil crear negocios para los propios ciudadanos y para las compañias extranjeras, ha tenido lugar un importante aumento del número de empresas. Incluso aquellas empresas estatales que no han experimentado grandes cambios tienen que operar en un entorno más competitivo, marcado por una mayor implicación en mercados internacionales y por la propia competencia en los mercados nacionales. Por todo ello, los países más avanzados del Este de Europa van integrándose efectivamente en el escenario europeo (y mundial) y sus relaciones económicas se vinculan progresivamente al mercado, aunque las consecuencias de este cambio (tanto a nivel estructural como de comportamiento) son todavía difíciles de evaluar.

Junto a estas transformaciones han tenido también lugar cambios paralelos en las políticas públicas y, por supuesto, en la política medioambiental. La mayoría de los países de la región, en los años que siguieron al colapso del antiguo régimen, tomaron medidas para elevar el status de sus administraciones medioambientales (creando organismos nacionales de rango ministerial donde no existían) y llevaron a cabo diversos programas con el objetivo de fortalecer las leyes y regulaciones en este área. Ha habido dos aspectos de particular importancia en este proceso: en primer lugar, se ha producido un endurecimiento de la postura de las agencias regulatorias, las cuales han adquirido mayor capacidad para perseguir sus objetivos medioambientales como resultado de un aumento de su status administrativo y de una reducción de las interferencias externas en sus esferas de responsabilidad; en segundo lugar, se ha llevado a cabo un incremento sustancial del nivel de multas y de cánones recaudados en conexión con la contaminación y el uso de recursos medioambientales - los cánones por contaminación atmosférica en Polonia aumentaron por 10 en términos reales entre 1989 y 1991 (MANSER, 1993), y algo similar parece haber ocurrido en la República Checa (CERna y Tosovska, 1991)-. En Polonia los gravámenes a las emisiones de $\mathrm{SO} 2$ están a un nivel de $60 / 80 \$$ por tonelada y son, por lo tanto, los más altos del mundo aunque sigan estando sustancialmente por debajo del coste de descontaminación a través de tecnologías de desulfurización.

Dadas las raíces sistémicas del fracaso de la política conservacionista en el Este de Europa existe una expectación comprensible en la región según la cual la transición dual, hacia el sistema político democrático y el sistema económico capitalista, producirá un 'dividendo medioambiental' sustancial con relativa independencia de las acciones específicas para fortalecer las leyes e instituciones de protección medioambiental ${ }^{6}$. Sería ridículo negar que esta idea tiene una cierta validez pues, de forma clara, el sistema económico capitalista occidental, junto con el sistema político democrático, tiene un mejor record en la 
protección del medio ambiente que los sistemas que dominaron hasta hace poco en el Este de Europa. Sin embargo, habría que incorporar aquí una importante cualificación: No es del sistema económico capitalista per se del que provendrán los beneficios medioambientales. El rápido crecimiento económico en España desde comienzos de los años 60 (como en muchos otros países anteriormente) estuvo acompanado de una pequeña o nula consideración por el medio ambiente y provocó, consiguientemente, una seria degradación del mismo. De hecho, las dinámicas económicas y políticas del régimen franquista exhiben algunas similitudes con las de los sistemas estalinistas del Este en relación a la prioridad adscrita a los objetivos productivistas (y al aumento del bienestar material) como medio de compensar la falta de democracia política. Además, el capitalismo democrático tiene muchas variantes y existen grandes diferencias en cuanto a su capacidad institucional para dar prioridad a los objetivos medioambientales y llevar a cabo políticas conservacionistas rigurosas. Es muy necesario, por lo tanto, prestar atención al diseño concreto de las políticas públicas y de las instituciones para la protección medioambiental pues el hecho de que el concepto de déficit de implementación se ajuste $\tan$ bien al Este de Europa no debe hacer olvidar que originalmente fue acuñado para describir la actuación de los países occidentales.

\section{Tendencias medioambientales en la Europa del Este y lecciones de la experiencia occidental}

El proceso de cambio económico en el Este de Europa tiene ya más de cinco años, ¿es entonces posible identificar algún tipo de tendencia que indique cómo será el estado del medio ambiente en un futuro próximo? Sin duda alguna, se puede afirmar ya que algunas de las áreas más afectadas por la contaminación, como la de la Alta Silesia, han experimentado una considerable mejora medioambiental. Las estadísticas nacionales reflejan claramente esta situación: En la República Checa, entre 1989 y 1992, las emisiones de SO2 descendieron aproximadamente un $23 \%$, al tiempo que las emisiones sólidas lo hacían en un 26\%. Las estadísticas de Polonia sugieren una tendencia aún más pronunciada pues las emisiones industriales de SO2 han bajado el $33 \%$, y las gaseosas un $40 \%$, mientras que la deposición de polvo se ha reducido en un 55\%.

Parte de esta mejora es debida indudablemente a una regulación más efectiva que ha promovido inversiones en equipos de descontaminación y una mejor gestión de los impactos medioambientales. Al mismo tiempo, y a pesar de que algunos especialistas sugirieron que el cambio en el comportamiento de las empresas en cuanto a la adopción de estrategias positivas para el medio ambiente sería relativamente lento, parece que según datos recientes (PINTO et al., 1993), y observación de primera mano, muchas empresas están siendo más flexibles en sus respuestas de lo que se podía prever. Sin embargo, otra parte de esta mejora es simplemente el resultado de un declive del ritmo de producción industrial asociado al proceso de cambio económico en la mayoría de los países del Este: El producto industrial bajó aproximadamente un $37 \%$ en la República Checa, y un $31 \%$ en Polonia en 1992 en comparación con 1989. Esto sugiere que el progreso medioambiental checo puede ser explicado más adecuadamente como resultado del descenso del producto industrial pues, entre otras cosas, el aumento de los cánones por las emisiones atmosféricas está siendo llevado a cabo gradualmente y sólo ha comenzado a ser realmente efectivo en 1993. Las estadísticas industriales de Polonia (que recogen cifras acerca del volumen de contaminación eliminada así como emitida) permiten extraer una imagen más sutil que muestra que la cantidad de contaminantes se redujó ampliamente en línea con la caída de la producción (30\% para las emisiones de polvo y $34 \%$ para las de gases) aunque, al mismo tiempo, tuvó lugar una descontaminación más eficiente que resultó en un disminución significativa de las emisiones: Cerca del 55\% de la reducción de las emisiones de polvo fue debida a una ralentización de la producción y un $45 \%$ a medidas más efectivas; para las emisiones atmosféricas, el $87 \%$ puede explicarse por el declive del ritmo industrial y cerca de un $13 \%$ por la mejora en la descontaminación (calculado a partir de Rocznik Statystyczny Przemyslu - Yearbbook of Industrial Statistics, 1993). Este análisis sugiere que hay diferencias significativas entre la situación de Polonia y la de la República Checa, pues el primer país está consiguiendo reducciones de las emisiones atmosféricas más significativas que el segundo, aunque éstas estén principalmente basadas en medidas correctoras (end-of-pipe) y concentradas en la deposición de polvo, con una eliminación mucho menos notoria de otros contaminantes (una pauta que también se aplica a la política medioambiental anterior a 1989). La cuestión crucial es lo que sucederá una vez que las economías del Este de Europa recuperen sus anteriores pautas de crecimiento (como ya sucede en el caso polaco): ¿Conducirá esta recuperación a un freno en la mejora medioambiental? El dilema podría ser resumido de la siguiente forma: La situación medioambiental en el Este en el año 2.000 no será seguramente tan mala como la de 1989, pero ¿será mejor o peor que la de mediados de los años 90 ?

La respuesta a esta pregunta no es simplemente de tipo predictivo sino que está determinada por variables políticas y, sobre todo, por la capacidad de los gobiernos de Europa del 
Este de desarrollar y aplicar efectivamente políticas medioambientales dirigidas principalmente al sector de la industria pesada. Teniendo en cuenta los contextos políticos específicos en el Este de Europa, ¿qué tiene la experiencia Occidental en cuanto a política medioambiental $\longrightarrow$, más concretamente, política atmosférica- que enseñar a la Oriental?

\subsection{Experiencias de política atmosférica en el Occidente}

Si se examinan a nivel comparado los datos sobre contaminación atmosférica puede afirmarse que la política de calidad del aire es, sin duda, una de las de mayor éxito dentro del campo medioambiental (o una de las que exhibe un menor grado de fracaso).

\begin{tabular}{|l|c|c|c|c|c|c|}
\hline \multicolumn{7}{|c|}{ Emisiones de SOx, NOx, y particulas de fuentes no móviles } \\
(en 1.000 toneladas)
\end{tabular}

Fuente: OECD, 1993; MOPT, 1985.

Una de las causas de este éxito ha sido indudablemente la preocupación generada por la contaminación transfronteriza y la lluvia ácida que, ya en los años 60 , provoca serios daños en los bosques nórdicos. Más adelante, la atención que se concedió en Alemania a este mismo problema conduciría a la aprobación de un estricto decreto sobre grandes plantas de combustión en 1983. La aproximación política que se seguía en este decreto, y que ponía el énfasis en la necesidad de controlar las emisiones ${ }^{7}$ de las grandes centrales eléctricas así como en el mantenimiento y la mejora de los estándares de inmisión o de calidad ambiental ${ }^{8}$, fue rápidamente incorporada por otros países que pertenecían al grupo de los más avanzados en cuanto a protección medioambiental. El decreto alemán también influyó en la Unión Europea que, en 1988, aprobó una directiva sobre grandes instalaciones de combustión. Esta nueva tendencia en la política atmosférica europea estuvo acompañada de difíciles negociaciones entre, principalmente, la UE y Gran Bretaña. Este país, que tradicionalmente había practicado una política de 'chimeneas altas' para dispersar sus contaminantes, se oponía a la adopción de los costosos estándares alemanes de protección atmosférica. Además, el Sur de Europa (y de forma muy visible España) defendía el 'derecho a contaminar más' como consecuencia, supuestamente ineludible, de una política económica que intentaría reducir la diferencia de bienestar material existente entre el Sur y el Norte en la UE.

\section{España: Una politica atmosférica sin participación social y a remolque de la Uniôn Europea}

En España el punto de partida de la política de calidad del aire es la Ley de Protección del Ambiente Atmosférico de 1972 ?.
Esta ley, que parte de una descripción relativamente optimista del estado del medio ambiente en España - según el texto, "no se han alcanzado aún niveles intolerables de degradación del medio ambiente salvo en casos muy excepcionales" (en GraU, 1988: 155)-, pone el énfasis en la fijación de niveles de inmisión a través de la introducción de una nueva figura: La zona de atmósfera contaminada (ZAC) como área en la que, por sobrepasarse los límites establecidos de inmisión, se adopta un régimen especial de actuaciones de tipo básicamente correctivo dispersión de las emisiones - y coyuntural —uso transitorio de combustibles menos contaminantes-. A mediados de los años 70, y basándose en esta ley y en su decreto de desarrollo (D 833/75), comienzan a aplicarse los primeros planes atmosféricos en ciudades con graves problemas de contaminación. Este es el caso del Plan Piloto de Urgencia para Bilbao de 1975 cuya puesta en práctica fue deficiente a pesar de que sus medidas eran muy limitadas - la sustitución de fuel-oil con un contenido en azufre del $3 \%$ por otro de contenido 1,5\%- Curiosamente, la explicación que en aquel momento dio la administración sobre los fallos en la aplicación de los planes no fue en absoluto auto-exculpatoria: aLa concienciación en España de este problema ha surgido en un momento totalmente inoportuno" (CIMA, 1978: 634). Quedaba así patente, de algún modo, el escaso interés de las autoridades públicas, que aún perdura, por promover una rigurosa política de calidad del aire (PÉREZ DE GreGORIO, . 1989).

Desde mediados de los años 80 se asiste a dos fenómenos importantes en la política de protección atmosférica: Las Comunidades Autónomas (CC.AAs) comienzan a asumir competencias en esta política, y se inicia la transposición de la legislación 
medioambiental comunitaria al Derecho interno. La gestión de la política atmosférica por las CC.AAs se recoge principalmente en planes de saneamiento atmosférico que introducen una cierta perspectiva global pues abarcan tanto la instalación de redes de medición (para prevenir situaciones de alarma atmosférica) como la implantación de sistemas de eliminación de los residuos sólidos procedentes de las actividades de descontaminación en el aire. El Programa de 1987 de Vigilancia y Control de la Contaminación Atmosférica en Huelva constituye uno de los mejores ejemplos de esta estrategia global. Por su parte, la adopción de las directivas de la UE ha obligado a las autoridades a afrontar nuevos problemas. Así se ha producido, por ejemplo, un aumento del número de ZAC (ante los más estrictos niveles de inmisión europeos), y se ha comprobado la necesidad de extender las redes de medición para controlar una mayor variedad de contaminantes. En otras ocasiones España ha logrado plazos de tiempo más largos, así como ciertas exenciones, a la hora de cumplir determinadas obligaciones medioambientales. Este es el caso de la directiva 88/609 sobre grandes instalaciones de combustión en la que se ha conseguido no reducir el porcentaje de emisiones de SO2 en 1993 con respecto a 1980 (frente a una disminución del $40 \%$ que practicarán países como Alemania), reducirlo sólo en un 24\% en 1998 (frente al 60\% en el anterior país), y en un $37 \%$ en el año 2003 (frente al $70 \%$ ). Sin embargo, como estas excepciones son limitadas, la transposición de la política medioambiental comunitaria ha resultado en un esfuerzo de adaptación a los nuevos y más exigentes estándares de protección atmosférica de la $\mathrm{UE}^{10}$. Asimismo, la incorporación del corpus de directivas ha promovido ciertos cambios en una política que, tradicionalmente, había reaccionado de forma reactiva con respecto a los problemas medioambientales y a los desarrollos de la protección atmosférica en la esfera internacional. Uno de los cambios que se percibe tras la entrada de España en la UE es una menor reticencia por parte de las autoridades públicas con respecto a la participación de los actores sociales en la política medioambiental: $Y$ ello a pesar de que esta política ha sido generalmente decidida por el gobierno dentro de una pauta de relación no cooperativa (o, en algunos casos, de cooperación no institucionalizada) con la industria, y de relativa exclusión de otros intereses sociales (como las asociaciones ecologistas). Esta nueva aproximación se refleja, entre otras cosas, en la creación en 1994 del Consejo Nacional del Medio Ambiente como órgano consultivo que reúne a muy diversos grupos sociales. Los pactos medioambientales, llevados a cabo en áreas muy degradadas, que promueven el ajuste entre planes públicos y privados de descontaminación y establecen un diálogo regular (relativamente institucionalizado) entre los gobiernos regionales y las industrias, constituyen también otra prueba de este cambio en el estilo político. A pesar de todo ello, la influencia de los actores sociales en la política medioambiental sigue siendo poco rele- vante y así lo ha denunciado recientemente Greenpeace al abandonar el Consejo Nacional del Medio Ambiente.

\section{Gran Bretaña: práctica del principio contaminador-paga- dor y reticencia a la política atmosférica de la Unión $\mathbf{E u}$ - ropea.}

Los principios básicos de la política medioambiental británica han permanecido prácticamente inalterados desde finales del siglo XIX hasta los años 70 (KNÖPFel y WEIDNER, 1985). Un enfoque político cauteloso (wait-and-see approach) contrapuesto al principio preventivo, la búsqueda de evidencia científica en contra de una aproximación política proactiva, y la preocupación por los costes y la eficiencia económica en detrimento de una política a más largo plazo basada en la inversión en I+D ", han producido una política de control de la contaminación atmosférica relativamente endeble. La aproximación reactiva en la política atmosférica es coherente con unas condiciones climatológicas favorables de fuertes vientos que permiten conseguir, con relativa facilidad, estándares aceptables de calidad ambiental; las medidas de control de la emisión, por el contrario, son más costosas para el país. Esto explica que, al contrario que en la UE, en Gran Bretaña haya primado el enfoque de inmisión sobre el de emisión, y también que la contaminación transfronteriza no haya sido un tema importante hasta que el país fue acusado de ser uno de sus responsables.

El marco de la política atmosférica se ha basado en la existencia de un programa mínimo de control de la contaminación, elaborado a nivel central, que ha garantizado una gran autonomía al organismo regulatorio medioambiental: El Servicio de Inspectores de la Contaminación (Her Majesty's Inspectorate of Pollution, HMIP) ${ }^{12}$. Este organismo fija de forma autónoma límites de emisión no obligatorios y deja a las autoridades regionales escasas posibilidades de aplicar estándares más estrictos. La reticencia a imponer estándares obligatorios a nivel nacional se muestra, por ejemplo, en que hubo que esperar a 1986 para que se establecieran límites en las emisiones de $\mathrm{SO} 2$ y NOx procedentes de centrales térmicas ${ }^{13}$ ya que en éstas la práctica habitual había sido la dispersión de contaminantes a través de chimeneas cuya altura era negociada entre el HMIP y los propietarios de las instalaciones. Asimismo, antes de que la directiva 80/779 entrara en vigor, tampoco existían niveles obligatorios de inmisión a nivel nacional.

Uno de los principios más importantes dentro de la política atmosférica de Gran Bretaña, introducido en 1874, es el de los mejores medios practicables (best practicable means, BPM). Este principio señala que las emisiones deben ser reducidas al mínimo posible y que no deben ser toleradas si existe algún riesgo para la salud. El HMIP considera que éste se está aplicando correctamente cuando se cumplen los límites no obligatorios de emisión establecidos a nivel nacional. El BPM está vinculado a 
una aproximación política flexible y pragmática, acorde con un control de la contaminación atmosférica practicado de forma casuística (sobre una base individual), que permite una gran libertad a la hora de aplicar los niveles permisibles de contaminación. Esto ayudaria a explicar la existencia de una relación cooperativa entre los diferentes organismos públicos medioambientales y la industria: La persuasión y la cooperación son así los instrumentos políticos más comunes al tiempo que los casos de cierre de empresas por razones medioambientales son muy escasos. En este sentido, podría señalarse que una pauta de tipo clientelístico habría reemplazado a un enfoque basado en la estandarización legal. Además, en Gran Bretaña existe también una gran confianza en la autoregulación industrial y en el cumplimiento voluntario de las obligaciones legales. Esto es posible, entre otras razones, porque la sociedad exhibe en general un cierto respeto por la función pública, la comunidad empresarial suele mostrar una actitud cooperativa con las autoridades, y los grupos sociales no sospechan en principio del poder de la industria (VOGEL, 1986). Uno de los pocos asuntos que podría ocasionar problemas en la cooperación entre autoridades e industria sería el temor mostrado por esta última acerca de la total adhesión del gobierno con respecto al principio contaminador pagador que ha conducido, entre otras cosas, a que la ayuda pública medioambiental a las empresas no sea grande. Por ello, las decisiones en cuanto a inversión en tecnología de descontaminación, y las elecciones (trade-offs) entre factores medioambientales y no medioambientales, son dejadas al libre arbitrio de la industria y del mercado ${ }^{14}$.

Un aspecto distinto de esta relación entre el gobierno y la industria lo constituye el pequeño número de intereses no empresariales con voz en esta política. A mediados de los años 70 comenzó a cuestionarse la cooperación exclusiva entre los responsables medioambientales y las industrias y, como consecuencia de ello, se produjo una apertura parcial del proceso político a grupos locales. Sin embargo los intereses no industriales siguen teniendo una posición débil, al tiempo que los costes económicos que acompañan a los litigios han inhibido a las asociaciones ecologistas con respecto a la posibilidad de emprender acciones legales contra los responsables de la contaminación. Los procedimientos de tipo secretista con la sociedad son generalmente la norma, al tiempo que el acceso público a los datos medioambientales está aún pendiente de resolución. Por otro lado, los dos principales partidos del país (el Conservador y el Laborista) han exhibido tradicionalmente sólo diferencias ínfimas en cuanto a la política medioambiental, lo cual se traduce, entre otras cosas, en un importante apoyo bipartidista a las leyes aprobadas. Este apoyo explicaría no sólo la gran estabilidad de la política a lo largo del tiempo, sino también que los problemas ecológicos estén menos politizados, gocen de menor relevancia que en otros países, y que no hayan entrado en el debate político hasta finales de los años 80 . Sin embargo esto está cambiando y, en 1987, una encuesta mostraba que la mayoría de los ciudadanos quería medidas conservacionistas más estrictas: El único tema en el que la protección del medio ambiente aparecía en segundo lugar era con respecto al desempleo (WEIDNER, 1987). El mayor interés de la sociedad por el conservacionismo, así como la creciente influencia de la UE sobre las políticas medioambientales de sus Estados miembros, podría estar en la base de los siguientes cambios: El reciente fortalecimiento de la administración del medio ambiente ${ }^{15}$, la aprobación de un corpus de legislación ajustado a los requisitos de la UE, la sustitución del consentimiento informal entre gobierno e industria por certificados oficiales basados en líneas de acción establecidas a nivel nacional, y la mejora del acceso a la información medioambiental. Asimismo, en 1989 y 1990 se introdujeron importantes innovaciones a través del informe titulado 'Esta Herencia Común' (This Common Inberitance) y del Acta de Protección Medioambiental. De esta forma, la política de control de la contaminación está cada vez más integrada ${ }^{16}$, y tanto los controles como las multas son ahora más frecuentes. Se puede señalar, para concluir, que -Gran Bretaña está ahora comprometida en un proceso de adaptación a las presiones medioambientales de Europa y a las crecientes presiones nacionales, pero todavía afronta dificultades considerables con respecto a la implementación de las medidas" (BOEHMER-ChristIANSEN, 1992: 318).

\section{Alemania: una política atmosférica de cooperación con la industria y de impulso en la Unión Europea}

La primera vez que la protección medioambiental se convierte en asunto político de importancia en Alemania es en 1961 cuando el Partido Social-Demócrata (SPD) promueve una campaña electoral de tono conservacionista bajo el título de 'Cielo azul sobre el Ruhr'. Posteriormente, tras su llegada al poder en 1969 en coalición con el Partido Liberal (FDP), el partido aprobó un detallado programa medioambiental y puso en marcha una política conservacionista de gran envergadura. Esta política se ha mantenido, e incluso fortalecido, tras la victoria electoral del Partido Cristiano-Demócrata (CDU) en 1982.

La política medioambiental alemana ha estado tradicionalmente basada en un extenso y detallado corpus de leyes y reglamentos elaborados y aprobados a nivel federal. El ejemplo paradigmático de este tipo de regulación es la Ley Federal para la Protección del Aire (Bundesimissionsscbutzgesetz, BImSchG) de 1974, y su reglamento (TA Luft) del mismo año. Esta ley constituye, en términos comparativos, uno de los instrumentos más estrictos en la política de control de la contaminación atmosférica. La BImSchG pone el énfasis en el control de las inmisiones y permite a las regiones - los Länder han sido los principales actores en la aplicación de esta política- fijar 
estándares de calidad ambiental más estrictos si existen circunstancias locales que así lo requieran, o si llegan a acuerdos voluntarios con las industrias. Por lo tanto, las autoridades regionales han tenido un considerable margen de maniobra que explica que el gobierno federal se resistiera, al principio, a imponer regulaciones uniformes sobre las emisiones. Sin embargo, el gobierno ha ganado gradualmente peso en esta política en detrimento de los Länder.

A pesar de que la BImSchG constituía a nivel internacional uno de los instrumentos más estrictos en la protección atmosférica, ofrecía considerables ventajas a los grandes agentes contaminantes (principalmente a las centrales de combustión antiguas) pues introducía diferentes criterios (como la practicabilidad económica, la proporcionalidad de la medida requerida, las posibilidades técnicas...) que debilitaban la exigencia de los objetivos medioambientales ${ }^{17}$. Desde mediados de los años 80 , sin embargo, esta política fue reforzada por medio del decreto sobre grandes plantas de combustión (GrossfeuerungsanlagenVerordnung). Este decreto fue el resultado de dos factores principales: El juicio de Voerde, por el cual un juez ordenó paralizar la ampliación de una central eléctrica ante la constatación de que la BImSchG no especificaba ni los niveles de emisión ni el estado de la técnica que debían aplicarse a las plantas de combustión; y la relevancia social que comienza a concederse al problema de la 'muerte de los bosques' (Waldsterben). Todo ello favorece un cambio en la orientación seguida hasta entonces en la política atmosférica en el sentido de darse ahora prioridad al control de los niveles de emisión estableciéndose, así, límites estrictos y uniformes a nivel nacional que reducen el margen de maniobra de las autoridades regionales. La nueva legislación establece asimismo criterios técnicos (la mejor tecnología disponible) que son más exigentes que el principio británico del BPM. Aunque el sector eléctrico y del carbón se resistió en un principio a estas novedades, terminó aceptándolas por distintas razones de las que cabe destacar dos: La perspectiva de que se impondrían obligaciones similares a las industrias europeas del ramo como consecuencia del proceso armonizador practicado por la UE y promovido por Alemania; y, más importante aún, la importante ayuda pública que, a diferencia del caso británico, el gobierno ha estado siempre dispuesto a aportar. Asimismo distintas instituciones privadas y públicas, así como diversos grupos de expertos, han favorecido una política medioambiental activa y tecnológicamente innovadora (BOehmer-Christiansen, 1992). Esto explicaría, por ejemplo, que Alemania sea el país dentro de la UE en el que la instalación de equipos de desulfurización está más extendida (más del 80\% del total de la capacidad europea) (OECD, 1993). La legitimidad que se atribuye a la tecnología como fuerza impulsora de la protección medioambiental es tal que el principio de anticipación/precaución (Vorsorge) -frente al principio reactivo que busca resolver problemas que van produciéndo- se- ha ido imponiéndose en el proceso político al tiempo que ha llegado a tener prioridad sobre los criterios de practicabilidad económica y de certeza científica.

Otro de los rasgos de la política de control de la contaminación atmosférica es que se ha caracterizado por promover una relación cooperativa entre los responsables públicos y los grupos industriales. De hecho, la industria está presente en el proceso político medioambiental desde sus inicios y las más importantes organizaciones económicas (por ejemplo, la Asociación Federal de la Industria Alemana, VDI) son enormemente influyentes en el mismo; esta influencia se demuestra en que, en algunas ocasiones, la VDI ha conseguido debilitar las exigencias conservacionistas contenidas en la regulación. La cooperación entre industria y gobierno queda perfectamente ejemplificada en la práctica de los acuerdos voluntarios (freiwillige Vereinbarungen). Estos acuerdos se desarrollan a lo largo de tres etapas: En la primera, el gobierno establece una serie de objetivos medioambientales (Zielfestlegung) que publica en el boletín oficial de la federación para que la industria pueda conocer sus intenciones; en la segunda, la industria puede elegir entre aceptarlos voluntariamente o esperar a que las autoridades los impongan a través de leyes o normas; en la tercera etapa, el gobierno organiza rondas negociadoras con aquellas industrias que han asumido los objetivos con el fin de llegar a compromisos voluntarios (orales o escritos, pero no vinculantes) para la realización de los mismos. A estos compromisos se les da posteriormente publicidad de forma que la opinión pública pueda actuar como garante de los mismos (AGUILAR, 1993a). La cooperación entre gobierno e industria se refuerza a partir de 1974 cuando se critica la poca eficacia de la aproximación tradicional de imposición regulativa (command-and-control), y se pone el énfasis en la auto-regulación industrial, el cumplimiento voluntario de la legislación, y los instrumentos económicos. Sin embargo, la aplicación del 'gobierno privado' de la industria no ha producido los resultados esperados. Este relativo fracaso, junto a la creciente sensibilidad conservacionista de la sociedad y los triunfos electorales de las listas verdes desde finales de los años 70, ha fortalecido la crítica acerca de un estilo político que es cooperativo exclusivamente en relación a los intereses económicos. A pesar de ello, sólo recientemente distintos grupos sociales han comenzado a ser incorporados de forma efectiva al proceso político medioambiental.

\section{Conclusiones}

La primera conclusión que podría extraerse de la experiencia occidental es que sólo la interacción entre sistema político democrático, sociedad sensibilizada por la protección del medio ambiente, y flexibilidad y capacidad de respuesta de los mecanis- 
mos de mercado, puede producir consecuencias medioambientales positivas. Junto a esto, los estudios sobre política atmosférica en los países occidentales han tendido a coincidir en puntos similares: Que la contribución más significativa a la mejora en la calidad del aire no siempre proviene de medidas políticas concretas sino de factores estructurales como los que afectan a cambios en la política energética, o al ritmo de producción industrial en tiempos de crisis económica; que los programas políticos relativamente simples, gestionados de forma centralizada, y dirigidos al control del contenido en azufre de distintos combustibles suelen tener efectos más positivos que los programas complejos de control de las emisiones; que el énfasis de los gobiernos en la actividad legislativa, y en la sustitución de regulaciones antiguas por otras nuevas, puede desviar la atención de un tema más importante como es el de hacer cumplir la legislación ya existente (enforcement); que la eficacia de la política no parece depender de la introducción de cláusulas que favorezcan la participación pública (aunque sí se puede hablar de una cierta relación entre política rigurosa y sensibilidad medioambiental social); y que el presupuesto asignado al organismo público encargado de la protección medioambiental no es el factor clave en el éxito de la política y sí los gastos en los que incurren aquellos sujetos que están sometidos a la regulación (las industrias) (KNÖPfEl Y WEIDNER, 1982, 1983, 1985, 1986; PORTNEY, 1991). Se podría así terminar diciendo, de forma resumida, que las medidas políticas simples y directas que se vinculan a (y refuerzan) tendencias estructurales, y se centran en los programas de cumplimiento y no en los de renovación legislativa, tienen más posibilidades de producir resultados positivos rápidos y de menor coste para la protección medioambiental ${ }^{18}$. Esta es una lección relevante para el Este de Europa como región en la que los recursos económicos y de tiempo son escasos.
- Este artículo es resultado de un proyecto ya finalizado de la Unión Europea (ACE-92-0256-R).

* Universidad de Salamanca.

** Universidad de Paisley.

1 Esta hipótesis sostendría, en última instancia, que la política pública determina la forma de hacer política dentro de la misma (las 'policies' condicionarian las 'politics').

2 En 1992, por ejemplo, la Agencia de Protección Medioambiental de Estados Unidos organizó en Budapest uno de sus Talleres internacionales de cumplimiento (international enforcement workshops) con este objetivo. Este proceso de transmisión de 'expertise medioambiental ha conducido a que algunos disenos políticos del Este se asemejen a una curiosa mezcla internacional en la que se combinan los estándares alemanes, los conceptos políticos norteamericanos, y las tradiciones administrativas locales.

${ }^{3}$ Estos programas corrieron, en la mayoría de los casos, una suerte paralela a los programas de reforma económica. Así Hungria, el país con mayor éxito con respecto a la innovación económica, exhibe también los mejores resultados en cuanto al control de sus actividades industriales más insensibles con el medio ambiente. No hay más que analizar los datos que siguen (que muestran el nivel relativamente alto, pero en descenso, de emisiones de $\mathrm{SO} 2$, y los grados de eficiencia energética) para comprobar esto.

4 Esta ansiedad por formar parte de la comunidad internacional dedicada a asuntos medioambientales contribuyó, de forma importante, a la desintegración de los sistemas políticos estalinistas. Muchos especialistas del Este experimentaron una gran frustración ante la incapacidad de sus gobiernos por llevar a cabo compromisos políticos y, como resultado, se concentraron en la organización de protestas conservacionistas que, en ocasiones, se vincularon a otros tipos de disidencia politica. El ejemplo más claro de esta disidencia se encuentra en Bulgaria: En este país los principales grupos de oposición se agruparon bajo el nombre de 'Ecoglasnost', al tiempo que la crisis política que condujo al colapso del régimen en 1989 fue provocada por protestas públicas contra la decisión del gobierno de acoger una conferencia internacional sobre el medio ambiente.

' Las principales fuentes de contaminación en el Este han estado asociadas a la industria pesada. Los datos de Polonia muestran, por ejemplo, que estos procesos industriales eran directamente responsables de casi la mitad de las emisiones de particulas (la principal causa de problemas de salud vinculada a la contaminación atmosférica) y de una gran proporción de emisiones de NOx, al tiempo que la fuente más importante de emisiones de $\mathrm{SO} 2$ es el sector eléctrico.

${ }^{6}$ Esto ha conducido a algunos políticos a cuestionar abiertamente el papel del estado en la protección medioambiental. Asi se muestra en unas recientes declaraciones, de 1994, del Primer Ministro checo Vaclav Klaus: -La propiedad privada (y la defensa de la misma) es la clave de cualquier solución del problema ecológico... Algunos ecologistas nos dicen que los propietarios privados no pueden ver más allá de sus narices y que sólo están maximizando 'los beneficios del dia'... y que por lo tanto es necesario que el estado entre en este juego y 'represente el futuro'. Pero esto es un gran error. Que un funcionario estatal pueda tener un interés a más largo plazo que un propietario (que tiene sus herederos) no parece que haya ocurrido históricamente. El Estado socialista y sus burócratas no demostraron nada de esto y ningún estado tiende a ser mucho mejor. Por lo tanto no confiemos en que quizás el estado (y sus consejeros con la mejor de las intenciones) pueda operar mejor que el mercado.

7 La emisión es definida por la legislación medioambiental española como •la cuantía de cada contaminante vertida sistemáticamente a la atmósfera en un período determinado, medida en las unidades de aplicación que corresponda a cada uno de ellos- (en Grau, 1988: 159). De forma resumida, la emisión es la contaminación que se mide a la salida del foco emisor.

${ }^{8}$ La inmisión se define como los limites máximos tolerables de presencia en la atmósfera de cada contaminante, aislado o asociado con otros en su caso• (en Grau, 1988: 159). De esta forma, los niveles de inmisión se miden en una zona territorial y son el resultado de la suma de las distintas emisiones dentro de la misma.

9 En 1993 se inicia la reforma de la ley de Protección del Ambiente Atmosférico. Como principales puntos de la misma habría que señalar la introducción de la figura de responsabilidad civil -los agentes que desarrollen actividades contaminantes tendrán que indemnizar a los periudicados por las mismas-, y la obligación por parte de las actividades catalogadas como potencialmente contaminantes de realizar auditorías medioambientales si quieren obtener ayudas económicas y fiscales de la administración (AguILAR, 1993b).

${ }^{10}$ Este esfuerzo explicaría la aprobación, en 1990, del Programa de Creación de una Base Energética y Tecnológica Medioambiental. Este programa del Ministerio de Industria es uno de los pocos que promueve una política de descontaminación preventiva mediante el fomento de tecnologías limpias, y la fijación de niveles de contaminación más estrictos que los existentes en la legislación vigente.

"El énfasis en la eficiencia económica ha conducido a defender la necesidad de tolerar efectos perjudiciales para el medio ambiente cuando el coste de eliminación de los mismos era extremadamente alto. Por esta razón, la tecnología de descontaminación atmosférica está relativamente poco desarrollada: Las centrales térmicas con equipos de desulfurización y desnitrogenización son escasas ya que, hasta hace poco tiempo, el lavado de carbón era prácticamente la única medida utilizada para reducir el $\mathrm{SO} 2$.

${ }^{12}$ El origen de esta institución se encuentra en el Servicio Alkali de Inspección (Alkali Inspectorate) creado en 1863. 
13 En el sector químico, sin embargo, sí era común la existencia de límites de emisión.

14 Es tema de discusión si este énfasis en el mercado se debe a un particular estilo político nacional enraizado en tradiciones culturales y acuerdos políticos estructurales- o a la llamada 'revolución thatcherista'. Para algunos autores, los consejeros y expertos medioambientales eran conscientes de los objetivos del gobierno conservador (privatización y des-regulación) y promovieron un enfoque basado en el mercado (BoeHMER-CHRISTIANSEN, 1992). Otra opinión sostendría, por el contrario, que la política de control de la contaminación ha sido poco influida por el thatcherismo.

is La Secretaría de Estado del Medio Ambiente ha conseguido poderes para establecer límites de emisión y estándares de calidad ambiental. Las autoridades locales, por su parte, tienen ahora también competencias para otorgar los permisos previos a la instalación de ciertas fuentes de emisión.

${ }^{16}$ Así, por ejemplo, el Servicio de Inspectores de la Contaminación -junto con la Autoridad Nacional de Cuencas Fluviales, y las 82 autoridades locales sobre residuos- será absorbido por la Agencia de Medio Ambiente (The Guardian, 1995).

17 Además, a mediados de los años 70 la política medioambiental perdió parte de su fuerza inicial por diferentes razones: La crisis económica, la resistencia de la industria, y el conflicto entre el Ministerio de Economía (cercano a los intereses industriales) y el Ministerio de Interior (responsable de la política medioambiental hasta 1986) (MüLler, 1986).

${ }^{18}$ Existen, sin embargo, diversas razones que permiten poner en duda esta conclusión relativamente pesimista acerca de los programas regulativos sofisticados. Los estudios sobre implementación fueron realizados a finales de los años 70 y principios de los 80 , cuando la mayoría de los programas de protección atmosférica llevaban en vigor menos de diez años. Este lapso de tiempo puede ser adecuado para evaluar otras políticas públicas, pero es claramente insuficiente en el caso de la politica medioambiental por tres razones básicas: Esta política, aunque no es completamente nueva, ha adquirido sólo recientemente cierta prioridad en determinadas agendas políticas; tiene un impacto directo en los intereses centrales de algunos actores poderosos como las multinacionales; y está asociada a procesos naturales (cambios metereológicos) y de largo plazo que son dificiles de controlar. Por lo tanto, es probable que los anteriores estudios hayan producido una imagen distorsionada al referirse a una etapa todavía temprana y formativa, cuando la resistencia al cambio era todavía alta (ni los tribunales habían elaborado una 'jurisprudencia conservacionista', ni la sensibilidad por el medio ambiente estaba muy extendida en la sociedad) y el potencial regulativo bajo. Sólo muy entrados los anos 80 , y debido tanto a un proceso de aprendizaje por parte de las agencias públicas como a un cambio de las actitudes sociales, comienza a aplicarse seriamente y a cumplirse (con diferentes grados) la regulación en este campo.

\section{Bibliografia}

- Aalders, M. (1993), -Regulation and In-Company Environmental Management in the Netherlands., Law and Policy, Vol. 15, April.

- Aguilar Fernández, S. (1993a), Políticas Medioambientales y Diseños Institucionales: la Comunidad Europea como Escenario de Negociación de una Nueva Area Política. Instituto Juan March de Estudios e Investigaciones. Madrid: Ed. Peninsular.

- Aguilar fernández, S. (1993b), Análisis Comparado de Políticas Medioambientales: El Control de la Contaminación Atmosférica en España y Alemania., Derecho Ambiental, nº 10.

- Aguilar Fernández, S. (1994) :Convergence in Environmental Policy?: The Resilience of National Institutional Designs in Spain and Germany, The Journal of Public Policy, Vol. 14, n²

- Ap SimOn, H. M. y WARREN, R. F. (1993), •Abatement Strategies for Sulphur Dioxide, and Analysis of the Role of Emissions from Central and Eastern Europe, Idojaras (Journal of the Hungarian Meteorological Service), Vol. 46, $n^{2} 4$.

- Boehmer-Christiansen, S. (1992), Anglo-German Contrasts in Environmental Policy-Making and their Impact in the Case of Acid Rain Abatement, International Environmental affairs, Vol. 4, Fall.

- Cerna, A. y Tosovska, E. (1992), Environmental Policy and Economic Reform. Prague: Institute of Economics, Academy of Sciences.

- CIMA (1978), Medio Ambiente en España 1978 (2 Edición). Madrid: MOPU

- Cummings, S. (1993), ·Polish Environmental Regulation: The State of Poland's Environment, Governmental Authorities, and Policy;, Suffolk Transnational Law Review, Vol. $16, \mathrm{n}^{2} 2$.

- Fisher, D. (1992), Paradise Deferred: Environmental Policy Making in Central and Eastern Europe. London.

- Fullenbach, J. (1988), European Environmental Policy: East and West. London.

- Grau Fernández, S. (comp) (1988), Legislación Ambiental. Madrid: BOE.

- HUCKE, J. y ULLMANN, A. (1980), Konfliktregelung zwischen Industriebetrieb und Vollzugsbehörde bei der Durchsetzung regulativer Politik, en R. Mayntz, (Hrsg.) Implementation politischer Programme. Königstein: Verlagsgruppe Athenäum.

- KNOPFEL, P. y WEIDNER, H. (1982), · Formulation and Implementation of Air Quality Control Programmes., Policy and Politics, Vol. 10, $\mathrm{n}^{2} 1$

- KNŐPFEL, P. y WEIDNER, H. (1983), .Implementing Air Quality Control Programs in Europe, en P. Downing, y K. Hanf (eds.) International Comparisons in implementing Pollution Laws. Boston: Kluwer-Nijhoff.
- KNÕPFEL, P. y WEIDNER, H. (1985), Luftreinbaltepolitik im internationalen Vergleich. Berlin: Sigma.

- KNÖPfel, P. y W/EIDNER, H (1986), Explaining Differences in the Performance of Clean Air Policies, Policy and Politics, vol. $14, n^{2} 1$.

- LowI, T. (1964), American Business, Public Policy, Case-Studies and Political Theory, World Politics, $\mathrm{n}^{2} 16$

- MANSER, R. (1993), The Squandered Dividend. London

- MAYNTZ et al., (1978), Vollzugsprobleme der Umweltpolitik. Kohlhammer

- MaYNTZ, R. (Hrsg.) (1980), Implementation politischer Programme. Königstein Verlagsgruppe Athenäum.

- MüLler, E. (1986), Innenwelt der Umwelpolitik. Opladen: Westdeutscher Verlag.

- NowICKI, M. (1993), AAir Pollution Problems in Poland.

- OECD (1993), Advanced Emission Controls for Power Plants. Paris: OECD.

- Pérez de Gregorio, J. J. (1989), :La Actuación del Ministerio Fiscal en la Investigación y Persecución de los Delitos Ecológicos;, en VV.AA Jornadas sobre el Delito Ecológico. Barcelona, 30 de noviembre y 1 de diciembre.

- Pinto, B. et al. (1993), -Transforming State Enterprises in Poland: Evidence on Adjustment by Manufacturing Firms, Brooking Papers on Economic Activity, 1.

- PorTney, P.R. (ed.) (1991), Public Policies for Environmental Protection. Washington: Resources for the Future

- Pratt, G.C. (1992), •Air Toxic Regulations in Four European Countries and the United States, International Environmental Affairs, Vol. 4, Spring.

- Randelzhofer, A. y WILKe, D. (1981), Die Duldung als Form flexiblen Verwaltungsbandelns. Berlin: Duncker \& Humblot.

- Rocznik Statystyczny Przemyslu, Yearbbook of Industrial Statistics (1993). Poland. - Richardson, J.J. (ed.) (1982), Policy Styles in Western Europe. London: George Allen \& Unwin.

- Stocock, B. (1992), The Eastern European Environmental Crisis: Its Extent, Impact and Solutions. London.

- The Guardian (periódico) (1995).

- VoGeL, D. (1986) National Styles of Regulation. Environmental Policy in Great Britain and the United States. Ithaca: Cornwell University Press.

- Weidner, H. (1987) Clean Air Policy in Great Britain. Berlin: Sigma.

- ZYuC, T. (1994) Internalizing Environmental Impacts of Industry in Poland: Preliminary Empirical Evidence. (mimeo). Warsaw: Ecological Economics Center, Economics Department, Warsaw University. 